\title{
Energy-Efficient Static Data Collector-based Scheme in Smart Cities
}

\author{
Adel D. Rajab* \\ College of Computer Science and Information Systems, Najran University, Najran, 61441, Saudi Arabia \\ *Corresponding Author: Adel D. Rajab. Email: adrajab@nu.edu.sa \\ Received: 02 December 2021; Accepted: 11 January 2022
}

\begin{abstract}
In the Internet of Things (IoT)-based smart city applications, employing the Data Collectors (DC) as the data brokers between the nodes and Base Station (BS) can be a promising solution to enhance the energy efficiency of energy-constrained IoT sensor nodes. There are several schemes that utilize mobile DCs to collect the data packets from sensor nodes. However, moving DCs along the hundreds of thousands of sensors sparsely distributed across a smart city is considered a design challenge in such schemes. Another concern lies in how these mobile DCs are being powered. Therefore, to overcome these limitations, we exploit multiple energy-limited Static Data Collectors (SDC) which are deployed at the locations optimized so that the energy consumption of both nodes and SDCs is minimized. Likewise, an Unmanned Aerial Vehicle (UAV)-enabled wireless power transfer is considered for sustaining the SDCs to avoid their energy depletion and data packet loss. The sustainable charging process operates periodically in each cycle so that the UAV flies from a charging station and after recharging the SDCs, it comes back to the station to be recharged. In this study, we formulate a problem to optimize the movement trajectory and charging time of UAV so that sustainable operation of SDCs during a cycle can be achieved. Unlike the prior studies, our proposed scheme determines the optimal trajectory and charging time at the beginning of each cycle which leads to increase accuracy in comparison with long-term optimization-based schemes. The outcomes of simulation experiments show that the proposed scheme achieves improved network performance in terms of data delivery ratio (12.5\%); system throughput (6.6\%); total energy consumption (59.19\%); and network lifetime $(58 \%)$ as compared to previous related works.
\end{abstract}

Keywords: Smart city; wireless sensor network; power constraint; energy efficiency

\section{Introduction}

The vast urbanization of population has provoked the need for smart cities [1-3]. A smart city refers to a set of Wireless Sensor Network (WSN)-based systems which leads to transformation of

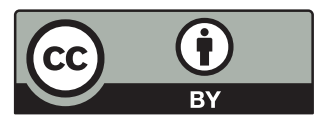

This work is licensed under a Creative Commons Attribution 4.0 International License, which permits unrestricted use, distribution, and reproduction in any medium, provided the original work is properly cited. 
traditional services to smart services such as smart health, smart homes, smart driving, and smart energy [4].

In a smart city, a WSN-based system consists of several battery-powered sensor nodes which generate different types of data from environment according to their defined functions and send to a control station [5]. Due to equipping the sensor nodes with limited energy resources, the energy efficiency is considered as one the most important challenges in WSNs [6,7]. There are several schemes which attempt to minimize the energy consumption of nodes and maximize the network lifetime [8-11].

In the last years, utilizing mobile agents (mobile data collector, mobile base station, mobile relay nodes) to collect data packets from sensor nodes is considered as an encouraging solution to reduce the transmission range of sensor nodes and their energy consumption which results in improving the energy efficiency [5,12-17]. In such applications, mobility can be achieved by attending the mobile elements with mobilizers for controlling their locations or they can be attached to transporters like vehicles, animals, robots. In mobile Data Collector (DC)-based schemes, DCs are responsible to move along rendezvous locations to collect data from sensor nodes and forward to a BS. The aim behind such schemes is to reduce the data transmission range of node and thereby reducing their energy consumption $[15,18-20]$.

In addition, there are several works that utilize mobile BS to achieve energy efficiency. Sink mobility can also improve the connectivity and reduce the collision, message loss, and latency. In [21], an optimized energy-efficient path planning strategy is presented to enhance the network lifetime and overcome the energy hole problem. In their study, multiple mobile sinks are utilized in order to enhance the energy efficiency of cluster heads. To this end, it is attempted to optimize the sojourn locations of mobile sink based on the weighted vertex cover problem. Furthermore, in [22], two mobile sinks are utilized to move along clusters and achieve balanced remaining lifetime of member nodes and cluster heads which leads to improve the coverage time and lifetime of the network. Moreover, an Energyefficient Mobile-sink Sojourn Location Optimization (EMSLO) scheme is presented in [23], which aims to maximize the coverage time and enhance the balanced energy consumption of nodes in a smart home. To this end, the sojourn locations of mobile sink is determined so that the variance energy of nodes is minimized.

Although, these approaches could remarkably enhance the network lifetime and energy efficiency, most of them consider unlimited energy supplies for these mobile devices. However, this assumption not only imposes high implementation cost on the network, but also it is not within a realistic network scenario. Moreover, in dense and large scale networks, multiple mobile devices need to be employed, which leads to extra network overhead due to optimizing their trajectory, sojourn location, sojourn time simultaneously. Therefore, in this paper, we propose an Energy Efficient Static Data Collectorbased (E2SDC) scheme that utilizes a few number rechargeable energy-limited Static Data Collectors (SDC), which are responsible to receive data from sensor nodes and forward to the base station. Likewise, to avoid the data packet loss which stems from energy depletion of SDCs, it is required to keep SDCs alive and recharge their batteries frequently.

In the past decade, Wireless Power Transfer (WPT) technologies has been integrated into rechargeable sensor networks which leads to maximize the operation time of rechargeable devices. There are several works which employ Wireless Mobile Chargers (WMC) to move along energy limited devices and recharge their batteries [24,25]. In most of such schemes, it is attempted to achieve sustainable operation of rechargeable devices via optimizing the trajectory and charging time of WMCs. However, in most of them, a long run prediction of trajectory, charging locations and charging time of mobile 
devices has been performed, which leads to reduce the accuracy. Therefore, in this work, it is attempted to optimize the trajectory and charging time of WMC during a charging cycle in such a way that keeping SDCs alive is guaranteed.

Among all WPT technologies, exploiting UAVs as energy transmitters for energy supplies [26-29], is most proper for smart cities environment due to existence of obstacles between rechargeable devices. Consequently, in this paper, we utilize an energy-limited UAV equipped with wireless charger which is able to recharge the SDCs. The main contributions of the proposed scheme are as follows:

- Unlike the previous data collector based schemes, which have utilized mobile data collectors, we employ few number static data collectors as the data transmission brokers between nodes and BS.

- In the proposed scheme, we consider the energy consumption of both sensor nodes and SDCs in optimizing the locations of SDCs.

- Unlike the prior WPT based schemes that attempt to enhance the operation time of sensor nodes, the aim behind our proposed work is to avoid the energy depletion of other service providers (static data collectors) via recharging their batteries permanently, which leads to reduce the data packet loss.

- In this paper, it is attempted to keep SDCs alive during a charging cycle using optimization of moving path and charging time of UAV. However, to the best of our knowledge, most of previous schemes attempt to achieve a long-run prediction of trajectory, charging locations and charging time of WMC, which leads to reduce optimization accuracy and results in decreased system throughput.

The remaining sections are organized as follows: Sections 1 and 2 give introduction and related work, respectively. The system model is explained in Section 3. The proposed scheme is given in Section 4. Simulation results is presented in Section 5. Section 6 gives the conclusion.

\section{Related Work}

In last decades, several schemes attempt to utilize data collectors as the data brokers between nodes and BS. In [30], the authors proposed an optimization framework for mobile data collection in energy harvesting WSNs. Authors employed mobile data collectors, named SenCar, which collects the data packets from specific sensor nodes and balances the energy consumption in the network. They first provide a case study in a solar-powered network to show the spatial-temporal energy consumption variation. Then, they improved a two-step data collection scheme. At the first step, some anchor nodes are selected as the sojourn positions of SenCars. At the second step, distributed algorithms are designed in order to maximize network utility.

In [31], the authors utilize multiple mobile DCs called mobile data patrons that are responsible to collect data from the sensor nodes in a sparse network and transfer to the BS, where, mobile data patrons require high amounts of energy to do transmission. In that work, authors attempt to improve the data transmission rate in such a way that the energy consumption of mobile data patrons can be minimized as much as possible. In [15], an efficient lifetime expansion method (LIEX) is presented, which utilized a mobile DC as a mediator between nodes and BS. In LIEX, the mobile DC is responsible to collect data packets from sensor nodes and then forward directly to the BS, which leads to reduce the data transmission range of sensor nodes and their energy consumption. In such scheme, the network area is divided into four logical sections and the mobile DC employs a learning automata to move towards the area center or to the network center at regular intervals of time. In 
[18], an Adaptive Hierarchical Data Dissemination (AHDD) mechanism is presented to overcome the fault-tolerance and energy hole problems. In AHDD, multiple mobile DCs are employed and their trajectory and sojourn locations are determined so that the energy consumption of sensor nodes will be balanced as much as possible.

An Intelligent Proficient Data Collection Approach (IPDCA) is proposed in [19] to transfer data packets from a large scale smart city to the control station. IPDCA employs multiple mobile DCs to read data from rendezvous points and then forward to a BS. Likewise, Bat algorithm is employed to optimize the movement trajectory of mobile DCs. An energy efficient procedure is described in [20] for a heterogeneous WSN, which utilized a mobile agent to reduce the energy consumption of nodes with lower remaining energy. In fact, the mobile agent is responsible to collect data from the nodes with higher energy consumption and then forward to a BS.

Furthermore, with the rapid expansion of charging technologies, a large number of studies have focused on charging algorithms for wireless chargers based WSNs. In [26], it is attempted to discover a Wireless Mobile Charger Excursion Optimization (WMCEO) in order to improve the operation time of nodes using a Wireless Mobile Charger (WMC). To this end, there are charging regions in the network and the mobile charger moves along these regions to recharge the nodes located at each region. In that work, first, the movement trajectory of charger is optimized so that the maximized energy efficiency can be achieved. Then, the charging time of charger is determined in such a way that the nodes belonging to the respective charging region is fully recharged. Furthermore, a Fair Energy Division Scheme (FEDS) is proposed in [27] aims to sustain the operation time of sensor nodes using two separate WMCs. The main objectives of FEDS are as follows: firstly, an energy share is assigned to each node, which is determined with respect to the energy consumption rate of the nodes and secondly, the energy levels and energy replenishment time of WMCs are taken into consideration in charging time optimization. In addition, Collaborative Mobile Sink Sojourn Time Optimization (CMS2TO) scheme is presented in [32] aims to maximize the network lifetime. To this end, CMS2TO attempts to determine the optimal sojourn location of mobile agent in such wise that balance operation time of nodes can be achieved. An energy efficient tour optimization of WMC based scheme is presented in [33] which aims to enhance the network performance. Their proposed scheme consists of two algorithms to optimize the movement path and charging time of WMC, where the aims behind these algorithms are to achieve balanced energy consumption and enhanced network lifetime, respectively.

\section{System Model and Assumptions}

The network model of the proposed scheme is depicted in Fig. 1. We consider a smart city with a set of $\mathrm{N}$ heterogeneous energy aware smart device with fixed locations, which are randomly deployed at the ground surface. The smart devices or the sensor nodes sense different types of data from a smart city environment and forward to a Base Station (BS). Moreover, in order to reduce the data transmission range of nodes and enhance the energy efficiency, we consider that there are $\mathrm{M}$ number of rechargeable energy limited SDCs as the brokers between nodes and the BS. It is assumed that all SDCs have different energy levels with respect to their power consumption rates

In this work, in order to avoid data packet loss, we consider a scenario that all SDCs need to be kept alive during cycle. Each SDC consumes energy for receiving data from sensor nodes and forwarding to the BS. In order to recharge the batteries of SDCs, we utilize a UAV equipped with Wireless Charger (WC) which moves along SDCs to transfer energy to their batteries. The power supply of UAV needs to be recharged at the end of a charging cycle after recharging the last SDC. To this aim, the UAV 
moves toward a charging station and stays there for a limited $\varphi$ time unit to be fully recharged. Fig. 2 shows a charging cycle in the proposed scheme.

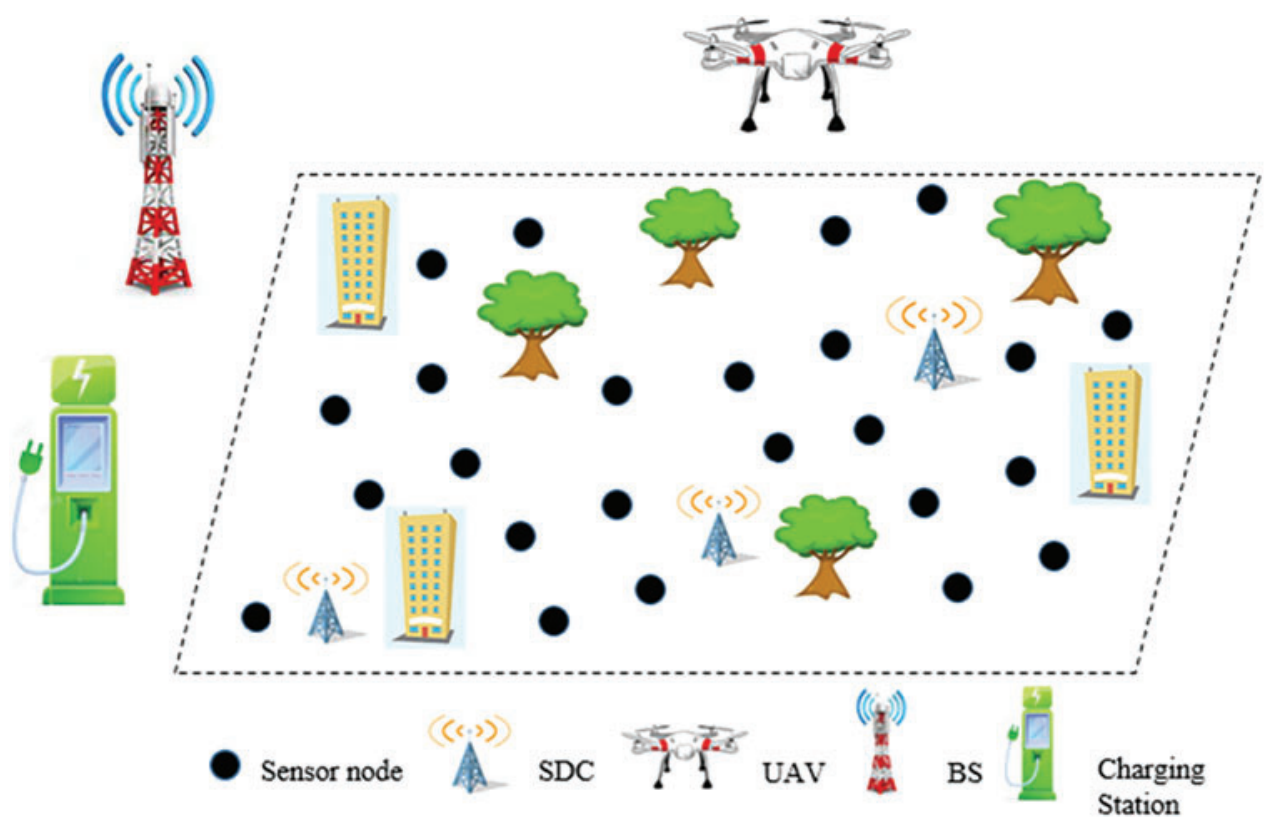

Figure 1: Network model

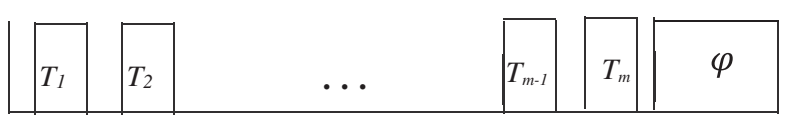

Figure 2: Charging cycle

Furthermore, we employ the power transmission model used in [34] to transfer energy from WMC to SDCs as follows:

$P_{r}=\frac{G_{s} G_{r} \eta P_{t}}{L_{P}}\left(\frac{\omega}{4 \pi(D+\beta)}\right)^{2}$

where the Pr and Pt denote the received and transmitted energy level. Likewise, Gs and Gr are the energy sender and energy receiver, respectively. Likewise, D, $\eta$, LP and $\omega$ denote the distance between Gs and Gr, rectifier efficiency, the polarization loss, and the wavelength. Moreover, in short-distance transmissions, $\beta$ is used to adjust the Friis' free space equation [35]. Then, Eq. (1) can be rewritten as follows:

$P_{r}=\frac{\mu}{(D+\beta)^{2}}$

Where,

$\mu=\frac{G_{s} G_{r} \eta P_{t}}{L_{P}}\left(\frac{\omega}{4 \pi}\right)^{2}$

Moreover, based on [32], $\mu=4.32 \times 10^{-4}$ and $\beta=0.2316$. 


\section{Energy-Efficient Static Data Collector-based Scheme}

In this work, to reduce the data transmission range of nodes and their energy consumption, $M$ number of SDCs are utilized, which are responsible to receive the data packets from sensor nodes and transfer to the BS. Having said that, to enhance the efficiency of SDCs, they need to be located at the optimal positions. On the other hand, as the employed SDCs are equipped with limited power supplies, to reduce the data packet loss, it is required to keep them alive as much as possible. To this end, a UAV is utilized which moves along SDCs to recharge their energy supplies. To achieve these objectives, we propose a E2SDC scheme which consists of two algorithms. In the first algorithm, the locations of SDCs are optimized so that the total energy consumption of nodes and SDCs will be minimized. In the second algorithm, the movement trajectory and the charging time of UAV is optimized so that the SDCs will not exhaust their energy during a charging cycle.

\subsection{Location Optimization of SDCs Mechanism}

As the energy consumption of power-consuming ground devices depends on their distances to their destinations, employing the brokers between nodes and BS leads to reduce their transmission range and energy consumption. To this end, in this work, we utilize few number of energy limited SDCs as the mediators between the nodes and BS. Likewise, in order to enhance the efficiency of SDCs, it is required to optimize the location of SDCs in the network. Therefore, in this section, we attempt to identify the optimal positions of SDCs in such a way that the energy consumption of nodes and SDCs is minimized as much as possible.

In this work, each node consume energy to transfer its sensed data to its respective SDC as follows:

$E_{t x}=l\left(E_{\text {elec }}+\propto d^{n}\right)$

In the above equation, Eelec, $\alpha$ and $\mathrm{n}$ are the electronic energy, the energy consumed for op-amp in data transmission and path loss exponent, respectively. $d$ denotes the distance between the node and respective SDC. The energy consumption of ith SDC to receive and transfer data packets to the BS can be formulated as follows:

$$
\begin{aligned}
& E_{r}(i)=\lambda(i) \times l\left(E_{\text {elec }}\right) \\
& E_{t r}(i)=\lambda(i) \times \beta \times l\left(E_{\text {elec }}+\alpha d_{t o B S}{ }^{n}\right)
\end{aligned}
$$

where $\lambda$ denotes the number of nodes which communicate with the ith SDC and $\beta$ is the compression ratio and is in the range $[1 / \lambda, 1]$ that $\beta=1 / \lambda$ is the perfect compression as the collected data are forwarded to the BS into a single packet. Then, the total energy consumption of ith SDC at each time unit is determined as follows:

$\operatorname{ESDC}(i)=E_{r}+E_{t r}$

Moreover, please let consider $\mathrm{L}=\{11,12, \ldots \ln \}$ is a set of locations of sensor nodes and $\mathrm{p}=\{\mathrm{p} 1$, $\mathrm{p} 2, \ldots, \mathrm{pm}\}$ is the locations of the SDCs. In this study, it is assumed that each node connects to the SDC with minimum distance. Then, in order to determine the respective SDC of each node, the distance between each node and the SDCs is needed to be separately determined.

$\forall x \in L, \forall y \in p(d(x, y)=$ distance $(x, y))$

$\operatorname{Res} \operatorname{SDC}(x)=\operatorname{SDC}$ with $\operatorname{Min}(d(x,:))$ 
where ResSDC denotes the respective SDC of $x$ th node. Then, the $\mathrm{p}$ is selected with joint consideration of the distance between nodes and SDCs and the energy consumption of SDCs. Therefore, the locations of SDCs will be optimized so that, firstly, the distance between nodes and SDCs is minimized; secondly, the SDCs consume least energy. Consequently, the problem is formulated as follows:

Minimize : $\eta_{1} \sum_{i=1}^{N}$ distance $(L(i), \operatorname{Res} S D C(i))+$

$\eta_{2} \sum_{i=1}^{M} \operatorname{ESDC}(i)$

where $\eta 1$ and $\eta 2$ are the weighting factors which specify the contribution percentage of the components in calculating the cost. After deploying the SDCs at the optimal locations, sensor nodes receive position information packets from SDCs and each node selects the nearest SDC to forward its sensed data.

\subsection{Sustainable Operation Time of SDCs-based Mechanism}

In this study, it is assumed that SDCs are equipped with limited battery supplies. Therefore, energy depletion of SDCs leads to stop its operation, which results in increased data packet loss and reduces network performance. Consequently, avoiding of energy exhaustion of SDCs is considered as a challenge in this work. To this end, we utilize a WC attached on a UAV to recharge the SDCs.

In the proposed scheme, the UAV is responsible for transferring energy to the batteries of SDCs during the charging cycles. In each charging cycle, UAV starts to move from a charging station, keeps move along SDCs, then lands and sojourns at the location of each SDC for a limited charging time to charge its battery and finally comes back to the charging station to harvest energy or recharge its power supply $(\varphi)$.

The main goal behind the proposed algorithm is to optimize the recharging order and the charging time of UAV at each SDC in such wise that the SDCs will not deplete their energy during the cycle. In the other word, the proposed algorithm guarantees the sustainable operation of SDCs during the respective charging cycle.

In this work, the recharging order of SDCs is determined so that the service quality is improved. Therefore, the SDCs with higher energy expenditure and lower remaining energy has higher priority to get service from UAV. To this end, UAV broadcasts request packets throughout the network and each SDC sends a remaining energy information (Rem) packet to the UAV. Then, UAV determines the order of SDCs according to their remaining energy level, as $\mathrm{O}=\{\mathrm{O} 1, \mathrm{O} 2, \ldots, \mathrm{Om}\}$.

Afterwards, the charging time at each SDC needs to be determined so that sustainable operation of SDCs will be guaranteed. To this end, UAV calculates the required energy level of each SDC according to received remaining energy information as follows:

$R E Q_{i}=$ Capacity - Rem $_{i}$

where, Capacity denotes the battery capacity of SDCs. Likewise, if we consider $\mathrm{T}=\{\mathrm{T} 1, \mathrm{~T} 2, \ldots, \mathrm{Tm}\}$ is the set of charging time of UAV at SDCs during a cycle, then, the Charging Time (CT) of UAV can be formulated as follows:

$C T=\sum_{i=1}^{m} T_{i}$

In addition, the travel time of UAV during a cycle can be formulated as follows:

$T T=T P \times$ Speed 
where, the speed denotes the velocity of UAV. Likewise, TP is the travelled path of UAV during a cycle, which is the summation of the Euclidean distances between SDCs and vertical distance between landing point at each SDC and flying altitude of UAV.

$T P=\sum_{i=1}^{m-1} \operatorname{distance}\left(P_{i}, P_{i+1}\right)+\operatorname{distance}\left(P_{C S}, P_{1}\right)+$

$\operatorname{distance}\left(P_{m}, P_{C S}\right)+(m \times 2) \times$ Height

where, $\mathrm{P}_{\mathrm{CS}}$ denotes the location of charging station and height is the flight altitude of UAV. Therefore, the cycle time can be determined using below Equation.

CycleTime $=T T+C T+\varphi$

In above Equation, $\varphi$ signifies the replenishing time of UAV at the charging station. Furthermore, the total energy consumption of a SDC during a cycle can be determined using following equation:

$E_{i}=\left(E S D C_{i} \times\right.$ CycleTime $)$

Accordingly, the remaining energy level of each SDC at the end of a cycle, will be:

$\operatorname{Rem}_{i}=\operatorname{Rem}_{i}-E_{i}+\left(T_{i} \times \lambda\right)$

where $\lambda$ indicates the received energy level at each time unit. In E2SDC scheme, it is attempted to transfer the required energy level of each SDC to its battery. Furthermore, as the main goal of the proposed algorithm is to guarantee that the SDCs will be kept alive, the remaining energy of SDCs need to be positive at the end of each cycle. Therefore, the following problem can be formulated.

$$
\begin{aligned}
\text { Maximize: } & \sum_{i=1}^{m} T_{i} \\
\text { suject to }: & T_{i}>0 \\
& T_{i} \leq R E Q_{i} \\
& \operatorname{Rem}_{\boldsymbol{i}}>\mathbf{0}
\end{aligned}
$$

\section{Simulation Results}

The simulation results to evaluate the E2SDC is presented in this section. The area of simulation map of the E2SDC scheme is approximately $200 \mathrm{~m} \times 200 \mathrm{~m}$ with $100 \sim 500$ sensor nodes and 2 6 SDCs and a static sink. In the methodology of the simulations, we start with evaluation of the impact of utilizing SDCs on the network performance against utilizing mobile data collector [15] and mobile sink [32]. Secondly, the impact of the introduced model for optimizing of the locations of SDCs on the network behavior is evaluated and compared with random deployment of SDC and position selection model proposed in [23]. Finally, the proposed UAV' tour optimization model is compared with related schemes proposed in $[26,27,33]$. The relevant parameters are shown in the Tab. 1.

\subsection{Impact of Utilizing SDCs}

Fig. 3 presents the data delivery ratio in three approaches, while increasing the number of sensors. As seen, in our proposed E2SDC, the data delivery ratio is higher than two other schemes. This is due to utilizing mobile devices in [8,9], which leads to frequent trajectory changes and high packet loss rate [35]. In addition, unlike two other related works, in our proposed scheme, increasing the number of nodes does not have impact on the network performance in terms of data delivery ratio. This is because, in mobile device based schemes, mobile sink or mobile DC needs to meet all nodes to serve 
them. Consequently, increasing the number of nodes leads to rise the movement path of mobile devices, which results in reduced data delivery ratio.

Table 1: Relevant parameters

\begin{tabular}{ll}
\hline Parameters & Value \\
\hline Number of nodes & $100 \sim 500$ \\
Network area & $200 \mathrm{~m} \times 200 \mathrm{~m}$ \\
Packet size & $128 \mathrm{bit}$ \\
Initial energy of nodes & $0.5 \mathrm{~J}$ \\
Energy dissipated in the op-amp & $0.0013 \mathrm{e}-12$ \\
Eelec & $50 \mathrm{e}-9$ \\
Number of SDCs & $2 \sim 6$ \\
Initial energy of SDC & $10 \mathrm{~J}$ \\
\hline
\end{tabular}

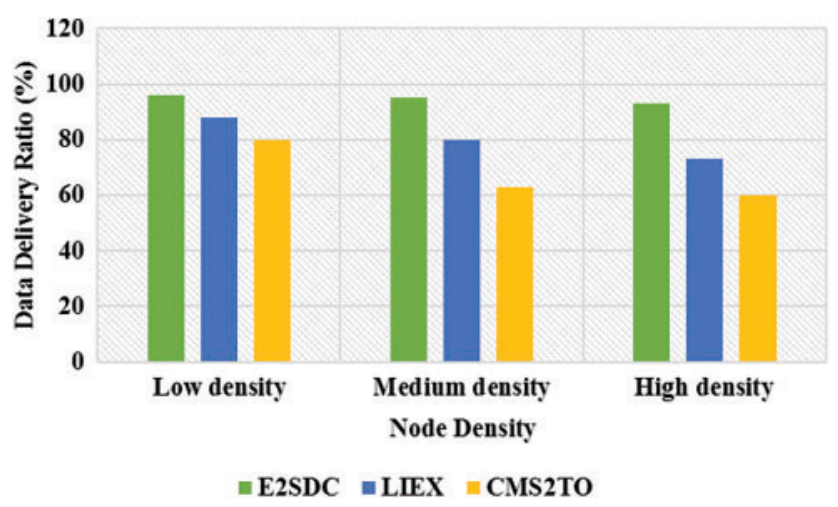

Figure 3: Data delivery ratio

Next, Fig. 4 evaluates the network performance in terms of end to end delay under varying number of nodes. As shown, the end-to-end delay of E2SDC, LIEX and CMS2TO schemes increased by incrementing the number of nodes. The reason behind this, higher the number of nodes in the network, incurs extra congestion in the network. However, E2SDC achieved lower average end-to-end delay compared to existing schemes thanks to utilizing multiple SDCs to collect data from sensor nodes.

The total energy consumption of nodes during a cycle is evaluated in Fig. 5 among different schemes as number of nodes increases. Obviously, higher number of nodes results in increasing the total energy consumption of network. E2SDC improves the performance as compared to existing schemes. The reason for the energy consumption reduction is to utilize the multiple SDCs, which leads to reduce the data transmission range of nodes and results in decreasing the energy consumption of nodes.

Moreover, the network lifetime is evaluated by considering number of rounds till first node depletes its energy supply. The purpose of evaluating the network lifetime is to show the improvement of E2SDC in saving the energy and prolonging the network operation time. Fig. 6 illustrates the network lifetime while varying number of nodes. Based on the simulation results, the proposed E2SDC scheme outperforms than other existing schemes as it has longer network lifetime at different node densities. Unlike the existing schemes, in E2SDC scheme, employing multiple SDCs leads to reduce 
the transmission range of nodes and decrease their energy consumption which results in enhanced network lifetime. Whereas, in LIEX and CMS2TO schemes, due to utilizing single mobile device, all sensor nodes have to send their data packet to the same destination even if they are located farther from mobile agent.

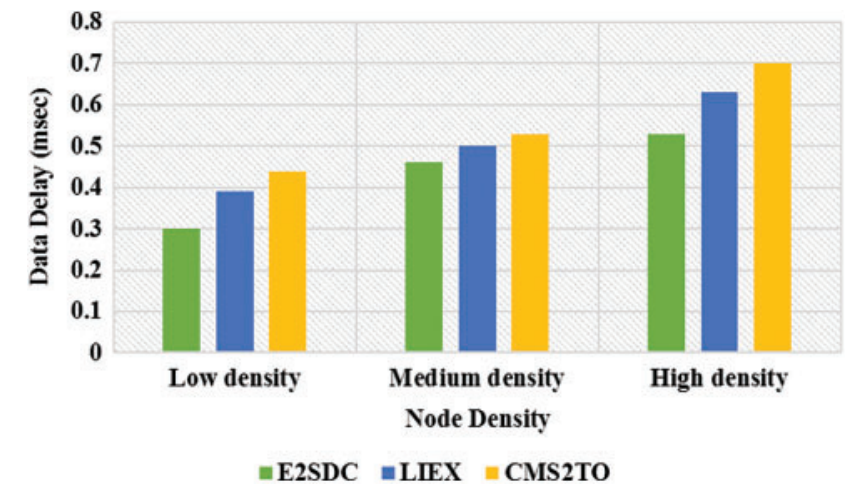

Figure 4: Data delay

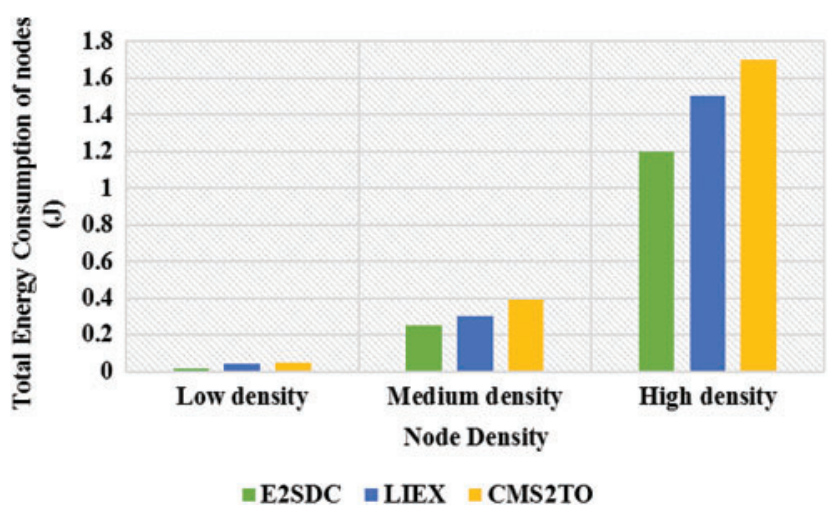

Figure 5: Energy consumption of sensor nodes

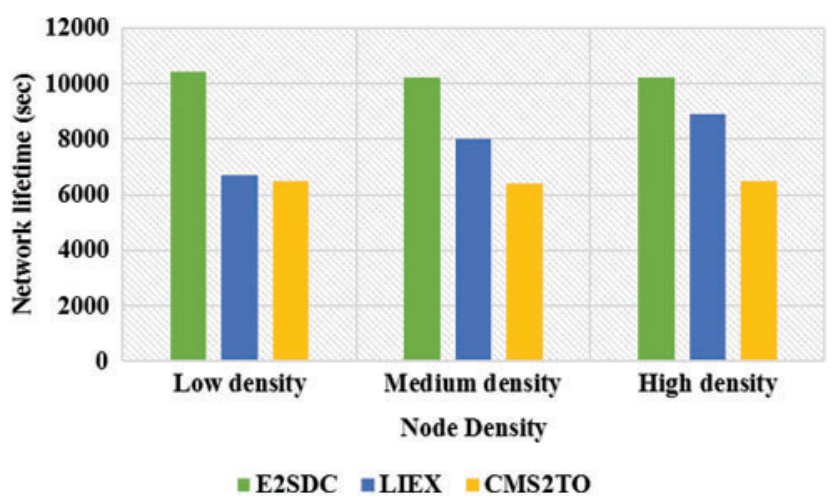

Figure 6: Network lifetime

Fig. 7 depicts the system throughput as the number of collected data per second. As evidenced, our proposed protocol outperforms mobile sink and mobile DC based schemes. This is due to utilizing 
mobile devices in previous schemes which leads to frequent trajectory changes and high packet loss ratio.

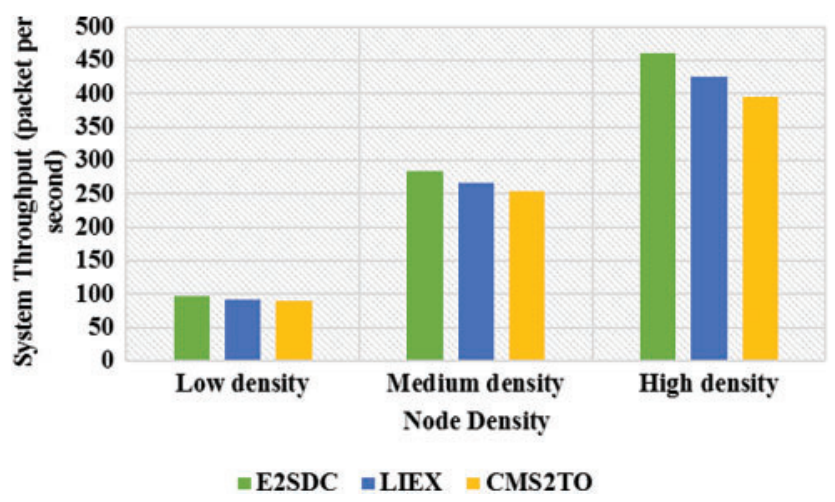

Figure 7: System throughput

The STD total energy consumption of SDCs is evaluated in Fig. 8 under different node distributions and number of SDCs. Increasing the number of SDCs clearly leads to increment in total energy consumption. However, as can be observed, the node distribution does not have impact on the energy consumption of SDCs. In addition, the effect of number of SDCs on the total energy consumption of sensor nodes are evaluated in Fig. 9. As evidenced, the energy consumption of nodes is reduced by incrementing the number of SDCs. This is due to increasing the number of SDCs which leads to reduce the transmission range of nodes and their energy consumption.

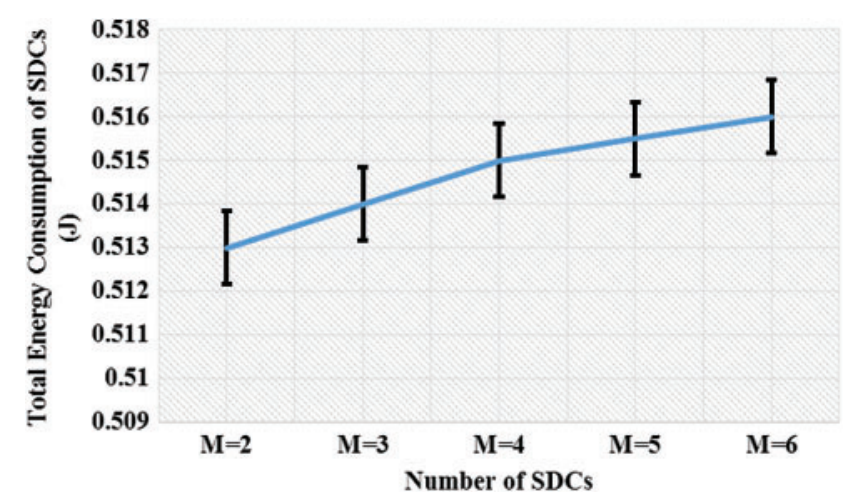

Figure 8: Total energy consumption of SDCs

\subsection{Location Optimization of SDCs}

In this sub-section, the impact of proposed location optimization of SDCs on the network performance is illustrated and compared with random deployment of SDCs and the location optimization scheme proposed in [23]. The performance comparison of E2SDC is estimated in terms of energy consumption of SDCs and sensor nodes.

Fig. 10 illustrates the energy consumption of SDCs in three approaches under different number of nodes. obviously, increasing the number of nodes leads to increase the number of collected data packets. 


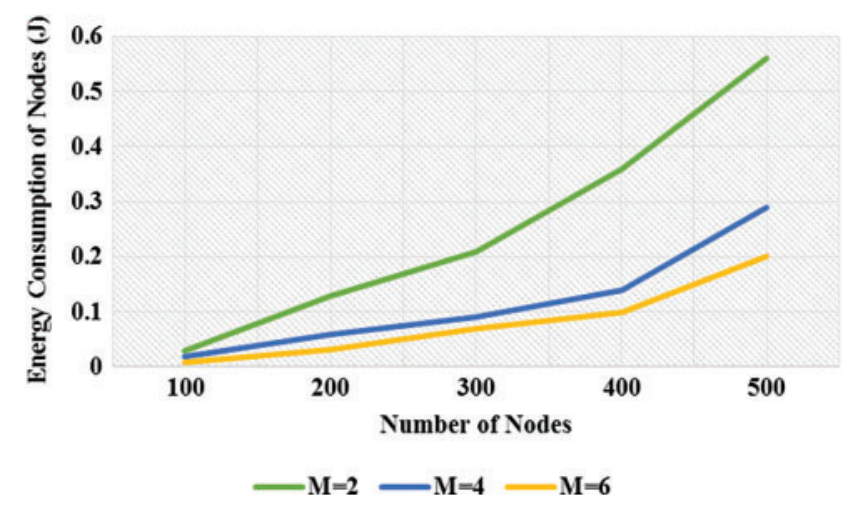

Figure 9: Energy consumption of nodes

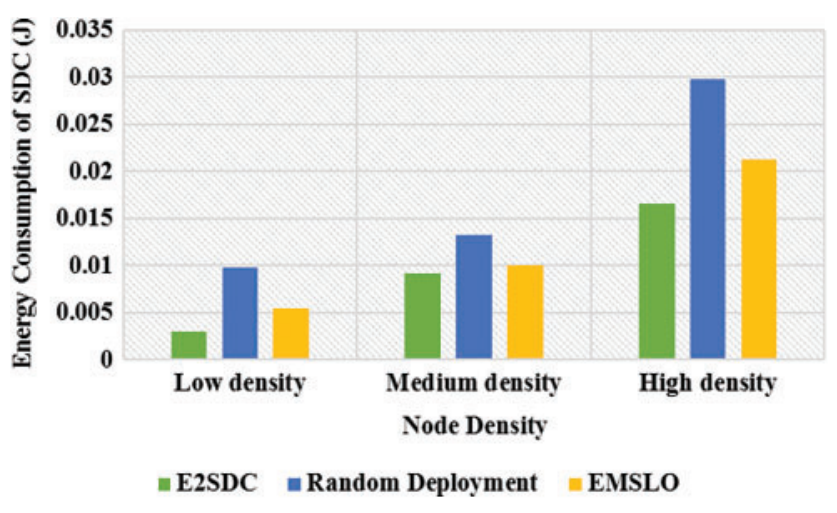

Figure 10: Energy consumption of SDCs

Likewise, as can be seen, in our proposed location optimization of SDCs mechanism, SDCs consume less energy compared with random deployment and EMSLO approaches. This is because, E2SDC scheme does not only consider the energy consumption of nodes but also takes into account the energy consumption of SDCs in optimizing the locations of SDCs. In addition, based on Fig. 11, in E2SDC, the sensor nodes consume less energy in comparison with two other mechanisms thanks to minimize the distance between nodes and SDCs using Eq. (9).

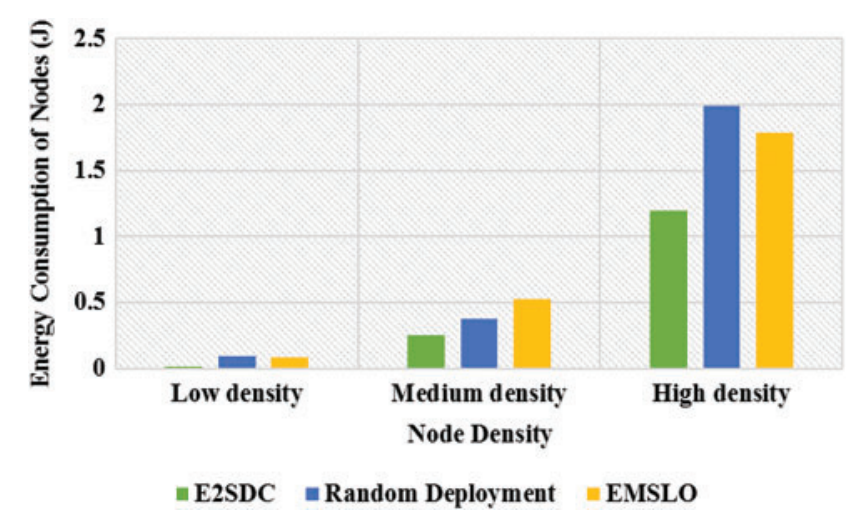

Figure 11: Energy consumption of sensor nodes 


\subsection{Sustainable Operation Time of SDCs}

In this sub-section, the proposed sustainable operation time of SDCs-based mechanism is evaluated in terms of computation time, charging time of UAV, and energy consumption ratio of SDCs. likewise, the movement path and charging time of UAV is determined by the approaches proposed in CTOWMC, WMCEO2, and FEDS schemes, inclusively. Then, the result of the proposed mechanism is compared with the relevant algorithms.

Fig. 12 depicts the computation time, defined as the running time of the mechanism, of the four different approaches under different node densities. The computational time of the proposed mechanism is clearly shorter than those of the three other schemes, which spend a remarkable length of time to determine the sojourn location of the charger. Our proposed scheme shortens the computational time because the UAV moves along a predefined path to recharge the SDCs.

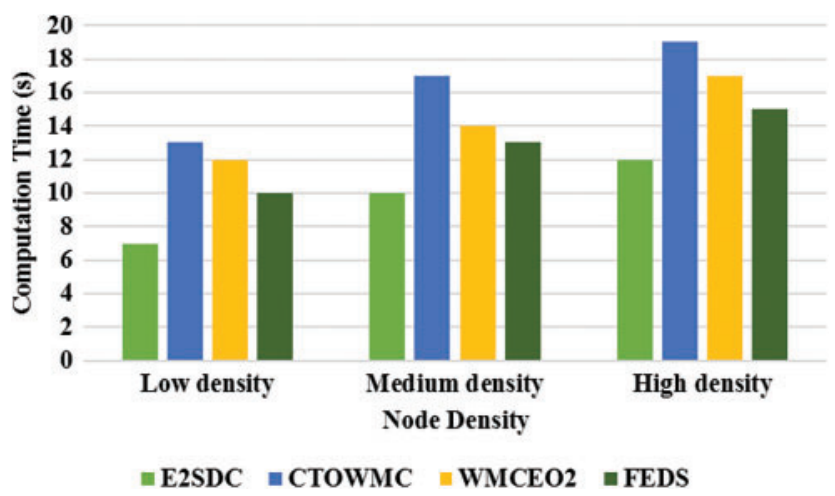

Figure 12: Computation time

Fig. 13 illustrates the charging time of UAV during a cycle under different number of nodes. As can be seen, our proposed approach outperforms other relevant schemes thanks to equation which defines a proper limitation for determining the charge time of UAV.

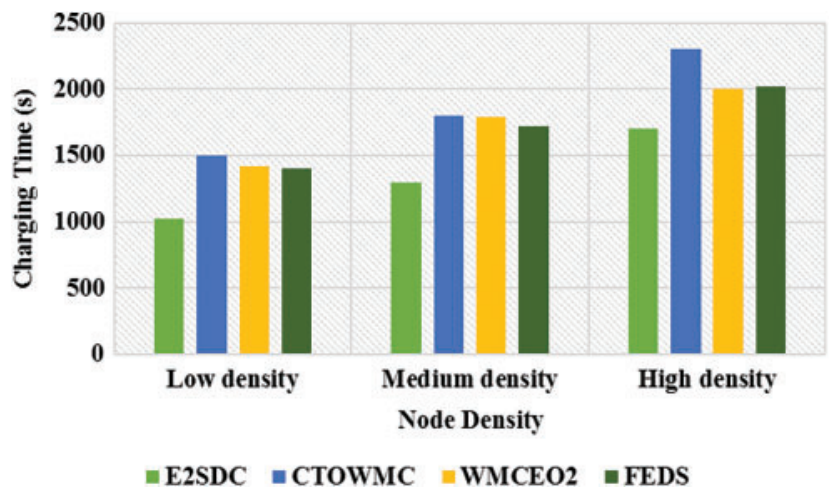

Figure 13: Charging time of UAV during a cycle

Fig. 14 depicts the energy consumption ratio of SDCs at the end of a cycle. Incrementing the number of nodes clearly leads to increase the data received by SDCs which results in arising the energy consumption of SDCs. Furthermore, as depicted, E2SDC and FEDS outperform CTOWMC and WMCEO2, as in both E2SDC and FEDS approaches, the remaining energy of SDCs has been taken into consideration in path optimization of UAV. 


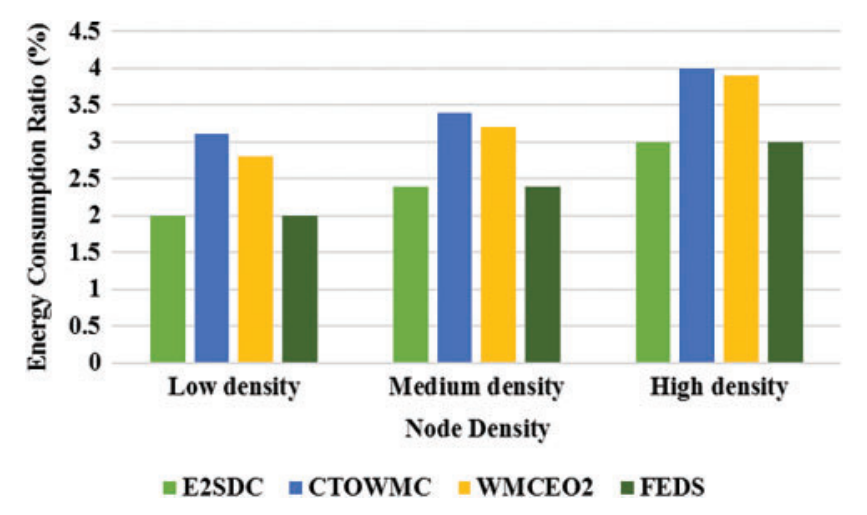

Figure 14: Energy consumption ratio of SDCs

\section{Conclusion}

In this paper, we present an efficient energy static data collector based scheme using UAV-enabled wireless power transfer in smart cities. We utilize multiple energy limited static data collectors which are responsible for reducing the data transmission range of nodes by receiving the data packets from sensor nodes and forwarding to the BS. First, we attempt to optimize the location of SDCs so that the minimized energy consumption of nodes and SDCs can be achieved. Unlike the prior studies, in the proposed scheme, the service providers (SDCs) are equipped with limited power supplies. Energy depletion of SDCs leads to data packet loss and reduce the network performance. Therefore, to address this limitation, we apply a UAV to recharge the batteries of SDCs frequently and sustain their operation time. To this end, it is attempted to optimize the trajectory and charging time of UAV during cycle in such a way that permanent operation time of SDCs would be guaranteed. Most of previous studies perform a long term prediction of trajectory and charging time of mobile agents, which leads to reduce the accuracy. Therefore, our proposed scheme repeats to do optimization of UAV's trajectory and charging time at the beginning of each cycle in such wise that the sustainable operation of SDCs can be guaranteed during a cycle. Finally, through simulations, we confirmed that the proposed scheme provides the perpetual operation of SDCs. Moreover, based on simulation results, it is observed that E2SDC outperforms other related works in terms of different evaluation metrics. The experimental results show that the proposed scheme achieves improved network performance in terms of data delivery ratio (12.5\%); system throughput (6.6\%); total energy consumption $(59.19 \%)$; and network lifetime (58\%) as compared to previous related works. Moreover, the proposed approach can be extended in the future works by considering the energy consumption of UAV in trajectory and charging time optimization. Additionally, in this study, we only developed the method for sustaining the SDCs operation. Accordingly, the future studies can further improve our method by recharging the sensor nodes by UAV to achieve perpetual operation of sensor nodes which results in permanent network lifetime.

Funding Statement: The authors received no specific funding for this study.

Conflicts of Interest: The authors declare that they have no conflicts of interest to report regarding the present study. 


\section{References}

[1] H. Sharma, A. Haque and F. Blaabjerg, "Machine learning in wireless sensor networks for smart cities: A survey," Electronics, vol. 10, no. 9, pp. 1012, 2021.

[2] A. Rehman, K. Haseeb, T. Saba and H. Kolivand, "M-SMDM: A model of security measures using green internet of things with cloud integrated data management for smart cities," Environmental Technology \& Innovation, vol. 24, pp. 101802, 2021.

[3] K. Haseeb, I. U. Din, A. Almogren, I. Ahmed and M. Guizani, "Intelligent and secure edge-enabled computing model for sustainable cities using green internet of things," Sustainable Cities and Society, vol. 68, pp. 102779, 2021.

[4] Q. Xie, K. Li, X. Tan, L. Han, W. Tang et al., "A secure and privacy-preserving authentication protocol for wireless sensor networks in smart city," EURASIP Journal on Wireless Communications and Networking, vol. 2021, no. 1, pp. 1-17, 2021.

[5] G. K. Ijemaru, K. L. M. Ang and J. K. Seng, "Mobile collectors for opportunistic internet of things in smart city environment with wireless power transfer," Electronics, vol. 10, no. 6, pp. 697, 2021.

[6] M. Shafiq, H. Ashraf, A. Ullah, M., Masud, M. Azeem et al., "Robust cluster-based routing protocol for IoT-assisted smart devices in WSN," Computers, Materials \& Continua, vol. 67, no. 3, pp. 3505-3521, 2021.

[7] J. J. Justus, M. Thirunavukkarasan, K. Dhayalini, G. Visalaxi, A. Khelifi et al., "Type II fuzzy logic based cluster head selection for wireless sensor network," Computers, Materials \& Continua, vol. 70, no. 1, pp. 801-816, 2022.

[8] K. Haseeb, S. Lee and G. Jeon, "EBDS: An energy-efficient big data-based secure framework using Internet of Things for green environment," Environmental Technology \& Innovation, vol. 20, pp. 101129, 2020.

[9] M. Alazab, K. Lakshmanna, T. Reddy, Q. V. Pham and P. K. R. Maddikunta, "Multi-objective cluster head selection using fitness averaged rider optimization algorithm for IoT networks in smart cities," Sustainable Energy Technologies and Assessments, vol. 43, pp. 100973, 2021.

[10] M. Sivaram, V. Porkodi, A. S. Mohammed and S. A. Karuppusam, "Improving energy efficiency in internet of things using artificial bee colony algorithm," Recent Patents on Engineering, vol. 15, no. 2, pp. 161-168, 2021.

[11] K. Haseeb, N. Abbas, M. Q. Saleem, O. E. Sheta, K. Awan et al., "RCER: Reliable cluster-based energyaware routing protocol for heterogeneous wireless sensor networks," PloS one, vol. 14, no. 9, pp. e0222009, 2019.

[12] A. Dande, S. Y. Chen, H. C. Keh, S. J. Yang and D. S. Roy, "Coverage-aware recharging scheduling using mobile charger in wireless sensor networks," IEEE Access, vol. 9, pp. 87318-87331, 2021.

[13] A. A. Kamble and B. Patil, "Systematic analysis and review of path optimization techniques in WSN with mobile sin," Computer Science Review, vol. 2021, no. 41, pp. 100412, 2021.

[14] P. Gupta, S. Tripathi and S. Singh, "RDA-BWO: Hybrid energy efficient data transfer and mobile sink location prediction in heterogeneous WSN," Wireless Networks, vol. 27, no. 7, pp. 4421-4440, 2021.

[15] M. Kamarei, A. Patooghy, Z. Shahsavari and M. J. Salehi, "Lifetime expansion in WSNs using mobile data collector: A learning automata approach," Journal of King Saud University-Computer and Information Sciences, vol. 32, no. 1, pp. 65-72, 2020.

[16] H. H. Choi and K. Lee, "Cooperative wireless power transfer for lifetime maximization in wireless multihop networks," IEEE Transactions on Vehicular Technology, vol. 70, no. 4, pp. 3984-3989, 2021.

[17] Y. Ren, A. Liu, X. Mao and F. Li, "An intelligent charging scheme maximizing the utility for rechargeable network in smart city," Pervasive and Mobile Computing, vol. 77, pp. 101457, 2021.

[18] N. Mazumdar, S. Roy, A. Nag and S. Nandi, "An adaptive hierarchical data dissemination mechanism for mobile data collector enabled dynamic wireless sensor network," Journal of Network and Computer Applications, vol. 186, pp. 103097, 2021.

[19] W. Osamy, A. M. Khedr, A. A. El-Sawy, A. Salim and D. Vijayan, "IPDCA: Intelligent proficient data collection approach for IoT-enabled wireless sensor networks in smart environments," Electronics, vol. 10, no. 9, pp. 997, 2021. 
[20] H. Singh, M. Bala and S. S. Bamber, "Augmenting network lifetime for heterogenous WSN assisted IoT using mobile agent," Wireless Networks, vol. 26, no. 8, pp. 5965-5979, 2020.

[21] B. R. AL-Kaseem, Z. K. Taha, S. W. Abdulmajeed and H. S. Al-Raweshidy, "Optimized energy eficient path planning strategy in WSN with multiple mobile sinks," IEEE Access, vol. 9, pp. 82833-82847, 2021.

[22] N. Gharaei, K. A. Bakar, S. Z. Hashim and A. H. Pourasl, "Inter-and intra-cluster movement of mobile sink algorithms for cluster-based networks to enhance the network lifetime," Ad Hoc Networks, vol. 85, pp. 60-70, 2019.

[23] N. Gharaei, S. J. Malebary, K. A. Bakar, S. Z. M. Hashim, S. A. Butt et al., "Energy-efficient mobile-sink sojourn location optimization scheme for consumer home networks," IEEE Access, vol. 7, pp. 112079112086, 2019.

[24] Y. Hu, X. Yuan, G. Zhang and A. Schmeink, "Sustainable wireless sensor networks with UAV-enabled wireless power transfer," IEEE Transactions on Vehicular Technology, vol. 70, no. 8, pp. 8050-8064, 2021.

[25] Y. Hu, Y.X. Yuan, J. Xu and A. Schmeink, "Optimal 1D trajectory design for UAV-enabled multiuser wireless power transfer," IEEE Transactions on Communications, vol. 67, no. 8, pp. 5674-5688, 2019.

[26] S. Malebary, "Wireless mobile charger excursion optimization algorithm in wireless rechargeable sensor networks," IEEE Sensors Journal, vol. 20, no. 22, pp. 13842-13848, 2020.

[27] A. O. Almagrabi, "Fair energy division scheme to permanentize the network operation for wireless rechargeable sensor networks," IEEE Access, vol. 8, pp. 178063-178072, 2020.

[28] J. Xu, Y. Zeng and R. Zhang, "UAV-Enabled wireless power transfer: Trajectory design and energy optimization,” IEEE Transactions on Wireless Communications, vol. 17, no. 8, pp. 5092-5106, 2018.

[29] X. Mo, Y. Huang and J. Xu, "Radio-map-based robust positioning optimization for UAV-enabled wireless power transfer," IEEE Wireless Communications Letters, vol. 9, no. 2, pp. 179-183, 2019.

[30] C. Wang, S. Guo and Y. Yang, "An optimization framework for mobile data collection in energy-harvesting wireless sensor networks," IEEE Transactions on Mobile Computing, vol. 15, no. 12, pp. 2969-2986, 2016.

[31] P. Prabu, A. N. Ahmed, K. Venkatachalam, S. Nalini and R. Manikandan, "Energy efficient data collection in sparse sensor networks using multiple mobile data patrons," Computers \& Electrical Engineering, vol. 87, pp. 106778, 2020.

[32] N. Gharaei, K. A. Bakar, S. Z. M. Hashim, A. H. Pourasl and S. A. Butt, "Collaborative mobile sink sojourn time optimization scheme for cluster-based wireless sensor networks," IEEE Sensors Journal, vol. 18, no. 16, pp. 6669-6676, 2018.

[33] N. Gharaei, Y. D. Al-Otaibi, S. A. Butt, S. J. Malebary, S. Rahim et al., "Energy-efficient tour optimization of wireless mobile chargers for rechargeable sensor networks," IEEE Systems Journal, vol. 15, no. 1, pp. 27-36, 2020.

[34] W. Na, J. Park, C. Lee, K. Park, J. Kim et al., "Energy-efficient mobile charging for wireless power transfer in internet of things networks," IEEE Internet of Things Journal, vol. 5, no. 1, pp. 79-92, 2017.

[35] S. He, J. Chen, F. Jiang, D. K. Yau, G. Xing et al., "Energy provisioning in wireless rechargeable sensor networks," IEEE Transactions on Mobile Computing, vol. 12, no. 10, pp. 1931-1942, 2012. 